\title{
Radiofrequency Thermo-Ablation of Morton's Neuroma: A Valid Minimally Invasive Treatment Procedure in Patients Resistant to Conservative Treatment
}

\author{
Ronconi Paolo ${ }^{1}$, Arcioni Roberto ${ }^{2}$, Baleanu Petre Mihai ${ }^{1}$ \\ ${ }^{1}$ Foot Medical Hospital, Rome, Italy; ${ }^{2}$ Pain Unit Sant’Andrea Hospital, University of Rome "La Sapienza”, Rome, Italy. \\ Email: pronconi52@gmail.com
}

Received October $8^{\text {th }}, 2013$; revised November $15^{\text {th }}, 2013$; accepted November $25^{\text {th }}, 2013$

Copyright (c) 2013 Ronconi Paolo et al. This is an open access article distributed under the Creative Commons Attribution License, which permits unrestricted use, distribution, and reproduction in any medium, provided the original work is properly cited. In accordance of the Creative Commons Attribution License all Copyrights @ 2013 are reserved for SCIRP and the owner of the intellectual property Ronconi Paolo et al. All Copyright (C) 2013 are guarded by law and by SCIRP as a guardian.

\begin{abstract}
Background: The authors present the personal results of the review of a group of cases treated for Morton's neuroma with continuous radiofrequency (CRF) thermo-ablation of the plantar intermetatarsal nerve. Methods: This retrospective review consisted of 29 patients treated between January 2008 and December 2011, with a minimum 1 year followup. Three patients underwent bilateral treatment and 10 patients underwent concomitant treatment procedures for associated foot disorders: hallux valgus (7), tailor's bunion (2) and hammertoe (5). The procedure was performed in a day hospital setting under direct fluoroscopic control. Results: The follow-up protocol used a visual analog scale (VAS) for pain $(0=$ no pain, $10=$ worst pain $)$ and a visual analog scale $(\mathrm{VAS})$ for patient satisfaction $(0=$ no satisfaction, $10=$ complete satisfaction). Success was defined by a decrease of 5 points in pain at 1 year after the treatment procedure. From these 29 cases, with a VAS pain score between severe and worst pain ever, treatment with CRF thermo-ablation at one year follow-up yielded these results: 14 cases with no pain (48\%), 7 cases with mild pain (24\%), 5 cases with moderate pain (17\%), 3 cases still with severe pain (10\%). The overall benefit was that $88 \%$ of the patients had significant pain improvements. From another point of view, 26 cases (89.6\%) had a decrease of the VAS score between 5 and 10 points and in only 3 cases (10.3\%) severe pain was reported, even though it was slightly diminished after the procedure. Conclusion: Continuous radiofrequency (CRF) thermo-ablation of Morton's neuroma (Entrapment) was a safe and minimally invasive surgical procedure which gave these patients great satisfactions and a rapid return to normal activity.
\end{abstract}

Keywords: Morton’s Neuroma; Continuous Radiofrequency (CRF) Thermo-Ablation

\section{Introduction}

Today, most physicians recognize that Morton's neuroma [1] is a nerve entrapment syndrome. This condition is exacerbated by the wearing of narrow shoes or the use of compressive support stockings. The characteristic pain is often described by the patient as: acute, intense, radiating into the toes, leg and often with strong burning sensation or local paresthesia. Local paresthesia of the 3rd and 4th toes is also common. The painful condition is frequently found in the third intermetatarsal space, less often in the second, rarely in the fourth and extremely rarely in the first intermetatarsal space [2]. The incidence of female: male ratio is 9:1 in our statistics, and it represents $25 \%$ $30 \%$ of overall forefoot complaints in our office [3].
The mean age of the patients was 45 years, with most cases diagnosed between the third and the sixth decade of life. The presence of the symptoms at rest or during the night should increase the suspicion of a neuropathy, prompting further clinical investigation. It is important to keep in mind that the Morton's neuroma could be an expression of a metabolic degenerative condition of the peripheral nerve, presenting as a clinical sign of a more complex neuropathy [2].

General diagnostic methods for Morton's neuroma include: a clinical exam which often reveals a positive Mulder's click [4], pain and digital paresthesia during the maneuver of placing medial to lateral compression on the forefoot while simultaneously applying plantar pres- sure 
on the affected intermetatarsal space ("squeeze test"), a high-resolution sonogram exam or MRI exam to visualize and confirm the presence of Morton's neuroma, and a gait analysis with the electronic baropodometric exam.

\section{Material and Methods}

We reviewed 32 feet in 29 patients affected by symptommatic entrapment, which did not respond to conservative treatment, between January 2008 and December 2011, in order to evaluate the efficacy of continuous radiofrequency (CRF) thermo-ablation-a minimally-invasive treatment of the plantar intermetatarsal nerve. The procedure was performed in a day hospital setting, and under direct fluoroscopic control. The follow-up was of minimum 12 months.

The diagnostic criterion for Morton's neuroma is clinical, with the very common description of the pain as: acute and extremely intense, with a burning sensation or "knife puncture" at the level of the third and fourth toe, radiating to the leg, and with the urgent necessity to liberate the foot from shoe compression. Our study followed best medical practices which included a clinical exam which revealed a positive Mulder's click [4], the "squeeze test", a high-resolution sonogram exam or MRI exam to visualize and confirm the presence of Morton's neuroma, and a gait analysis with the electronic baropodometric exam. All patients presented with clinical signs and underwent this evaluation. Differential diagnosis was made with other metatarsalgias, radiculopathies, tarsal tunnel and peripheral neuropathies because the subjects' symptoms did not correspond to the pathology of Morton's neuroma and they did not enter into the study group.

We hypothesized that the metatarsophalangeal pain with neuralgic characteristics frequently is caused by the compression of the branches of the plantar digital nerves on their course through the intermetatarsal space.

Once the correct diagnosis of Morton's neuroma was established, we proceeded with the following five-step therapeutic algorithm:

First step therapy: larger shoes, orthoses, local ice, massage and physical therapy, nonsteroid anti-inflammatory drugs (NSAIDs). Depending on the outcome for the patient, this step can last from 15 days to 2 months.

Second step therapy: corticosteroids plus local anesthetic injection $(40 \mathrm{mg} / \mathrm{ml}$. methylprednisolone acetate with 2 cc of $2 \%$ mepivacaine), 1 injection, eventually repeated after two weeks if the response is good after the first injection. This step is followed for 30 or 40 days to see if the injections are beneficial.

Third step therapy: Continuous radiofrequency (CRF) thermo-ablation of the intermetatarsal branches of the plantar nerve. After the intervention, a minimum of 4 - 6 months must pass in order to determine a successful outcome. If the intervention is not successful, it is attempted again or the fourth step is undertaken.

Fourth step therapy: surgical endoscopic decompression of the TIML (EDIN-Barrett technique) [5]. This surgery requires a minimum of 6 months in order to evaluate success.

Fifth step therapy: neuroma amputation with a dorsal longitudinal intermetatarsal approach.

Pain relief after local radiofrequency nerve ablation is thought to result from an increase in local temperature at the site of application around the electrode to the point of disruption of the tissue protein chains, which subsequently destroys the peripheral nerve endings, and the myelin sheaths, blocking only nociceptive input $[6,7]$. Other authors have hypothesized that pain relief after RF neurotomy results are not from the actual destruction of nerve tissue, but rather from strong electric fields induced by voltage fluctuations in the area of treatment $[8,9]$.

In this study we only considered patients treated with continuous radiofrequency (CRF). Continuous RF (CRF) describes a process whereby RF current is used to produce a thermal lesion in a target nerve, resulting in interruption of nociceptive afferent pathways [8]. The CRF produces a real nerve thermo-ablation in situ at a temperature of $75^{\circ} \mathrm{C}$ for 2 minutes with the probe positioned parallel and linear with the nerve, providing the destruction of the nerve fibers. Pulsed RF (PRF) delivers short bursts to a target nerve producing effects on signal transduction to reduce pain. These procedures do not produce a permanent nerve lesion [10]. The PRF, which reaches $42^{\circ} \mathrm{C}$, is applied perpendicular to the nerve and determines a partial and reversible lesion of the nerve fibers with the risk of recidivism in our experience. Even though these procedures are equally minimally invasive, we preferred a stable result as we obtained with the CRF method.

In our study we considered a group of 29 patients diagnosed with refractory Morton's neuroma who had undergone steps one and two of our treatment algorithm, between January 2008 and December 2011, with continuous radiofrequency (CRF) thermo-ablation of the intermetatarsal branches of the plantar nerve. Among the 29 patients, 27 were female and 2 were male. 3 patients were treated bilaterally. 4 patients were treated for 2nd and 3rd spaces at the same operative time. Patients' age ranged from 27 to 72 years, with the minimum follow-up period being 12 months.

All the patients were informed about the other surgical treatment options and they freely chose to attempt continuous radiofrequency thermo-ablation (CRF) therapy. 


\subsection{Surgical Technique}

In the operating room with the patient supine on the operating table the painful intermetatarsal area was marked and the sterile operating field was prepared. Under direct fluoroscopic control (Figures 1 and 2) the radiofrequency probe was introduced in the intermetatarsal space and under the transverse intermetatarsal ligament from an inter-digital approach and linear to the plantar intermetatarsal nerve (Figure 3). The motor stimulation through the probe was useful to control and avoid muscle lesion during the radiofrequency application. The application started when the patients informed the surgeon that the pain induced by the sensorial probe test was similar to the pain usually felt as a symptom. When the patient's answer was positive $1 \mathrm{cc}$ of $2 \%$ of mepivacaine was injected through the probe and the radiofrequency application started at a impedance value of 450 Ohms and 4.5 watts, for 2.5 minutes, producing a local increase in temperature to $75^{\circ} \mathrm{C}$. The procedure was repeated in 3 distinct points along the course of the intermetatarsal branch of the plantar nerve. Once the procedure was finished the probe was removed and the patient was discharged with a small bandage. Walking was permitted with a sport shoe with weight-bearing as tolerated. Rest at home for 24 - 48 hours prior to returning to work was

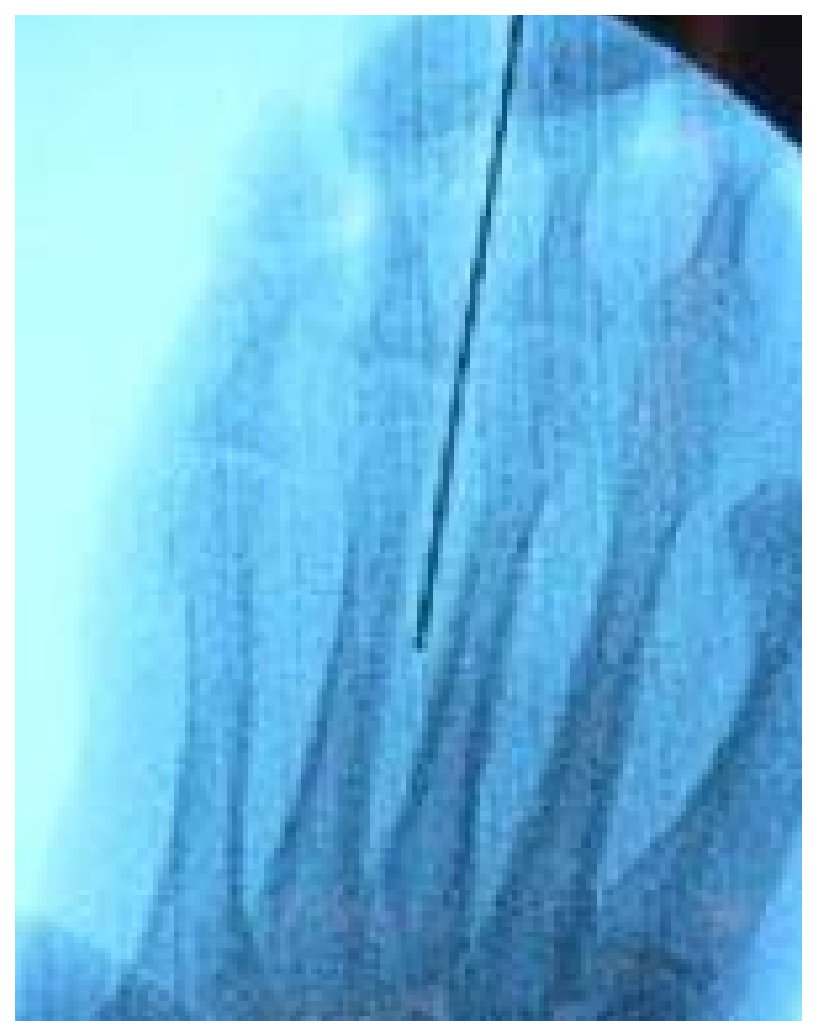

Figure 1. Anteroposterior radiograph showing probe inserted in the correct interspace.

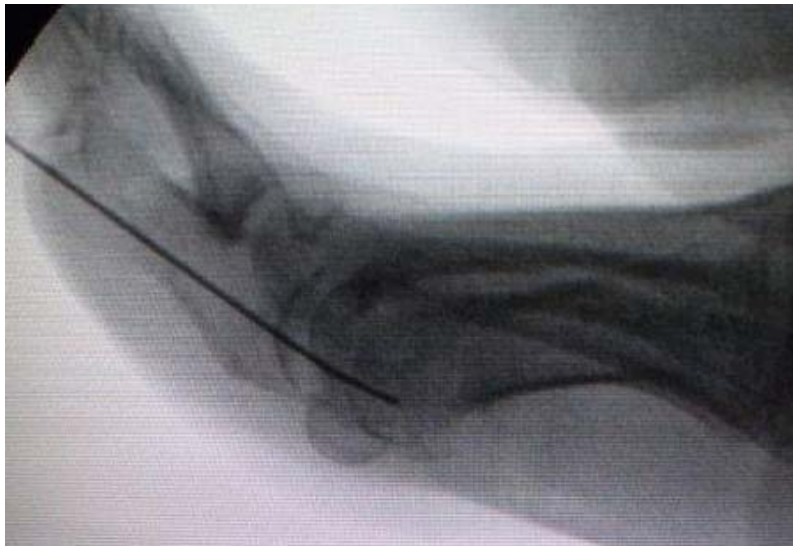

Figure 2. Lateral view.

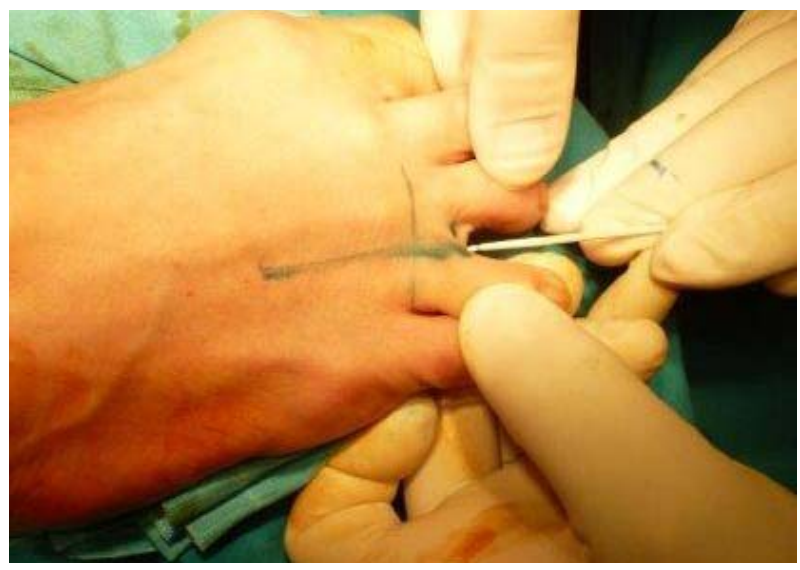

Figure 3. Probe inserted in the $3^{\circ}$ interspace.

recommended. During the first 1 - 3 days postoperatively the patient could use the NSAID of their choice for pain management. The routine follow-up was done at $24-48$ hours after the procedure, at 10 days, 20 days, 4 weeks, 3 months, 6 months and 12 months.

\subsection{Outcome Surveys}

Patient self-evaluation for pain and function was made by a linear VAS score for pain ranging from 0 (no pain) to 10 (worst pain) (Table 1).

Patient self-evaluation of satisfaction was made with VAS in linear fashion from 0 (completely unsatisfied) to 10 (completely satisfied).

\subsection{Final Results}

From the 29 cases with a VAS pain score between severe and worst pain ever, at one year follow-up after the treatment with CRF thermo-ablation of Morton's neuroma we obtained: 14 cases with no pain (48\%), 7 cases with mild pain (24\%), 5 cases with moderate pain (17\%), 3 cases with severe pain $(10 \%)$. These 5 cases with moderate 
Table 1. Vas pain score.

After treatment 1year follow-up

Before treatment

Final results: from 29 cases (100\%) with severe to worst pain ever, at one year followup after the treatment with CRF thermo-ablation of the Morton's neuroma we obtained: 14 cases no pain (48\%), 7 cases mild pain (24\%), 5 cases moderate pain (17\%), 3 cases severe pain (10\%). The overall benefit was $88 \%$ sensible improvement with a decrease of the VAS score between 5 to 10 points in 26 cases $(89,6 \%)$ and only 3 cases with severe pain but slightly diminished after the procedure.

\begin{tabular}{|c|c|c|c|c|c|c|c|c|c|c|c|}
\hline \multirow[b]{2}{*}{ SCORE } & \multirow{2}{*}{$\begin{array}{c}\text { No pain } \\
0\end{array}$} & \multicolumn{2}{|c|}{ Mild pain } & \multicolumn{3}{|c|}{ Moderate pain (discomforting) } & \multicolumn{2}{|c|}{ Severe pain (distressing) } & \multicolumn{2}{|c|}{ Very severe (horrible) } & \multirow{2}{*}{$\begin{array}{c}\text { Worst pain ever } \\
10\end{array}$} \\
\hline & & 1 & 2 & 3 & 4 & 5 & 6 & 7 & 8 & 9 & \\
\hline \multicolumn{12}{|l|}{ CASE } \\
\hline 1 & $\mathbf{0}$ & & & & & & & & 8 & & \\
\hline 2 & & 1 & & & & & & & & 9 & \\
\hline 3 & & & & & 4 & & & & & & 10 \\
\hline 4 & $\mathbf{0}$ & & & & & & & 7 & & & \\
\hline 5 & & & & 3 & & & & & & & 10 \\
\hline 6 & $\mathbf{0}$ & & & & & & & & 8 & & \\
\hline 7 & & 1 & & & & & & & & 9 & \\
\hline 8 & & & & & & & 6 & & & & 10 \\
\hline 9 & & 1 & & & & & & & & & 10 \\
\hline 10 & $\mathbf{0}$ & & & & & & & & & 9 & \\
\hline 11 & $\mathbf{0}$ & & & & & & & & & 9 & \\
\hline 12 & & 1 & & & & & & & 8 & & \\
\hline 13 & & & & 3 & & & & & & & 10 \\
\hline 14 & & & & & 4 & & & & & & 10 \\
\hline 15 & $\mathbf{0}$ & & & & & & & & & 9 & \\
\hline 16 & $\mathbf{0}$ & & & & & & & & & 9 & \\
\hline 17 & $\mathbf{0}$ & & & & & & & & & 9 & \\
\hline 18 & & 1 & & & & & & & & & 10 \\
\hline 19 & & & & 3 & & & & & & & 10 \\
\hline 20 & & & 2 & & & & & & & & 10 \\
\hline 21 & 0 & & & & & & & & & 9 & \\
\hline 22 & 0 & & & & & & & & & 9 & \\
\hline 23 & & 1 & & & & & & & & 9 & \\
\hline 24 & 0 & & & & & & & 7 & & & \\
\hline 25 & 0 & & & & & & & & 8 & & \\
\hline 26 & 0 & & & & & & & & 8 & & \\
\hline 27 & & & & & & & & & 8 & 9 & \\
\hline 28 & & & & & & & & 7 & & 9 & \\
\hline 29 & 0 & & & & & & & & & & 10 \\
\hline total & 14 & 6 & 1 & 3 & 2 & & 1 & $1 / 2$ & $1 / 5$ & 12 & 10 \\
\hline
\end{tabular}


pain and 3 cases with severe pain can be explained by an imperfect application of the technique. $88 \%$ of the patients treated had an overall benefit of pain improvement. 26 patients (89.6\%) had a decrease of the VAS score between 5 to 10 points and in only 3 (10.3\%) cases was there severe pain reported, even though slightly diminished, after the procedure, as showed in Graphs 1 and 2.

The 10 patients who underwent interventions for hallux valgus (7), Tailor's bunion (2) and hammertoe (5) in conjunction with CRF had a better overall result due to the improvement in biomechanical function. They had a total improvement as compared to the patients who underwent CRF only.

At the end of the study we also noticed that $40 \%$ of the patients still had some degree of footwear restriction, $90 \%$ had plantar and digital numbness at 3 months follow-up, corresponding to the treated intermetatarsal space and to the 3rd - 4th toes, but most patients were unaware of the sensory loss at 6 months follow-up. Wearing fashionable shoes for a long time during the day (6 to 8 hours) remained a problem for most patients even though they were happy with the treatment received.

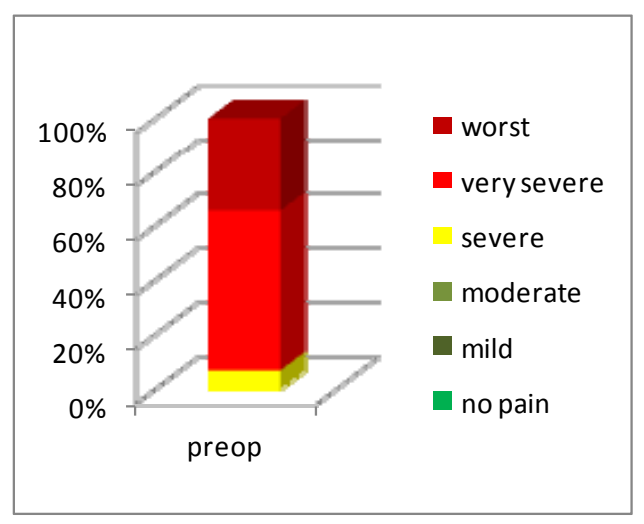

Graph 1. VAS pain score before treatment.

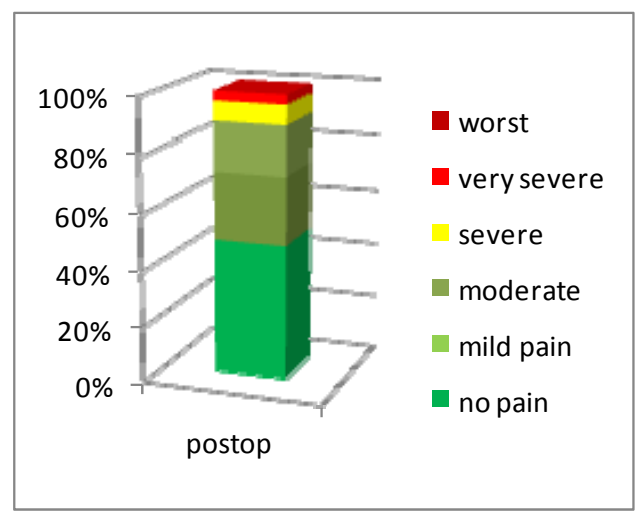

Graph 2. VAS pain score after treatment. No significant complications were encountered.
With comfortable shoes, overall satisfaction was excellent in 26 patients (89\%).

\section{Conclusion}

The results of our retrospective study indicate that the continuous radiofrequency (CRF) thermo-ablation of the intermetatarsal branches of the plantar nerve is a minimally-invasive day surgery procedure, effective and safe for the patient affected by symptomatic neuroma, and a third step therapy prior to other surgical and more invasive procedures because the resected nerve is always exposed to mechanical stress and it can be involved in a scar between skin and subcutaneous tissue, leading to formation of painful neuroma.

\section{Discussion}

Many treatments have been proposed for the treatment of neuromas, often conservative in nature and focusing on the inflammatory aspect of neuromas.

CRF is an innovative and rapid technique that can be done in ambulatory conditions without sutures and it gives the same percentage of positive outcomes as more invasive procedures and without serious complications. The procedure has minimal to no side effects or disability and is a very good third step therapy for patients hoping to avoid open surgery.

\section{REFERENCES}

[1] T. G. Morton, "A Peculiar and Painful Affection of the Fourth Metatarsophalangeal Articulation,” American Journal of the Medical Sciences, Vol. 71, No. 141, 1876, pp. 37-45.

http://dx.doi.org/10.1097/00000441-187601000-00002

[2] M. Frascarelli, M. Urciolo, P. Monachino, M. Baleanu and G. Favilli, "La Sindrome di Morton. (Valutazione Elettromiografica),” Chirurgia Del Piede, Vol. 22, No. 3, 1998, pp. 151-155.

[3] A. Viladot, "Metatarsalgia Due to Biomechanical Alterations of the Forefoot," Orthopedic Clinics of North America, Vol. 4, No. 1, 1973, pp. 165-178.

[4] J. D. Mulder, "The Causative Mechanism in Morton's Metatarsalgia," The Journal of Bone \& Joint Surgery British, Vol. 33, 1951, pp. 94-95.

[5] S. L. Barrett, E. Rabat, M. Buitrago, V. P. Rascon and P. D. Applegate, "Endoscopic Decompression of Intermetatarsal Nerve(EDIN) for the Treatment of Morton's Entrapment-Multicenter Retrospective Review," Open Journal of Orthopedics, Vol. 2, No. 2, 2012, pp. 19-24.

[6] E. R. Cosman Jr. and E. R. Cosman Sr., "Electric and Thermal Field Effects in Tissue around Radiofrequency Electrodes,” Pain Medicine, Vol. 6, No. 6, 2005, pp. 405424. http://dx.doi.org/10.1111/j.1526-4637.2005.00076.x

[7] L. Todorov, "Pulsed Radiofrequency of the Sural Nerve 
for the Treatment of Chronic Ankle Pain,” Pain Physician, Vol. 14, No. 3, 2011, pp. 301-304.

[8] J. Zundert, P. Raj, S. Erdine and M. van Kleef, “Application of Radiofrequency Treatment in Practical Pain Management: State of the Art," Pain Practice, Vol. 2, No. 3, 2002, pp. 269-278.

http://dx.doi.org/10.1046/j.1533-2500.2002.02036.x

[9] J. Van Zundert, A. J. de Louw, E. A. Joosten, A. G. Kessels, W. Honig, P. J. Dederen, et al., "Pulsed and Con- tinuous Radiofrequency Current Adjacent to the Cervical Dorsal Root Ganglion of the Rat Induces Late Cellular Activity in the Dorsal Horn,” Anesthesiology, Vol. 102, No. 1, 2005, pp. 125-131.

http://dx.doi.org/10.1097/00000542-200501000-00021

[10] W. Rea, S. Kapur and H. Mutagi, "Radiofrequency Therapies in Chronic Pain," Continuing Education in Anaesthesia, Critical Care \& Pain, Vol. 11, No. 2, 2011, pp. 35-38. http://dx.doi.org/10.1093/bjaceaccp/mkq057 